\title{
On infinite divisibility and embedding of probability measures on a locally compact Abelian group
}

\author{
By Herbert Heyer (Tübingen) and Gyula Pap (Debrecen)
}

\section{Introduction}

In the present note the authors supplement significant properties of infinitely divisible and embeddable probability measures on a locally compact Abelian group $G$. There are at least two versions of infinite divisibility appearing in the literature which deserve special attention, and the problem of embedding those measures leads directly to the study of continuous convolution semigroups on $G$. It is evident from the classical setup of $G=\mathbb{R}^{d}$ for $d \geqslant 1$ that in this context Gaussian semigroups and measures play a favorite rôle.

The main result of Section 3 concerns the representation of Gaussian measures in terms of their characteristics and their relationship to Gaussian measures in the sense of Parthasarathy. Section 6 and 7 deal with the embedding of infinitely divisible probability measures in the weak or strong sense respectively. We present direct proofs to more or less known statements, but stress the irreplacable hypothesis that the dual $\widehat{G}$ of $G$ is arcwise connected. In a concluding Section 8 we initiate the study of Gaussian and diffusion hemigroups on $G$ and their analysis, especially for 1-dimensional connected Abelian groups.

\section{Preliminaries}

Let $G$ be a locally compact group with identity element $e$. Let $\mathfrak{M}^{1}(G)$ denote the semigroup of probability measures on $G$. For every $x \in G, \varepsilon_{x}$ denotes the Dirac measure in $x$. For a compact subgroup $H$ of $G, \omega_{H}$ denotes the normalized Haar measure on $H$.

2.1 Definition. Let $H$ be a compact subgroup of $G$. A family $\left(\mu_{t}\right)_{t>0}$ in $\mathfrak{M}^{1}(G)$ (indexed by positive real numbers) is called an $\boldsymbol{H}$-continuous convolution semigroup in $\mathfrak{M}^{1}(G)$ if $\mu_{s} * \mu_{t}=\mu_{s+t}$ for all $s, t>0$ and $\lim _{t \downarrow 0} \mu_{t}=\omega_{H}$.

A family $\left(\mu_{t}\right)_{t>0}$ in $\mathfrak{M}^{1}(G)$ is called a continuous convolution semigroup in $\mathfrak{M}^{1}(G)$ if $\mu_{s} * \mu_{t}=\mu_{s+t}$ for all $s, t>0$ and the limit $\mu_{0}:=\lim _{t \downarrow 0} \mu_{t}$ exists in $\mathfrak{M}^{1}(G)$. The limit measure $\mu_{0}$ is an idempotent element of $\mathfrak{M}^{1}(G)$ and hence $\left(\mu_{t}\right)_{t>0}$ is an $H$-continuous convolution semigroup in $\mathfrak{M}^{1}(G)$ for some compact subgroup $H$ of $G$.

This research has been supported by the Deutsche Forschungsgemeinschaft and the Hungarian Academy of Sciences. 
2.2 Definition. A continuous convolution semigroup $\left(\nu_{t}\right)_{t>0}$ in $\mathfrak{M}^{1}(G)$ is called a Gaussian semigroup if

$$
\lim _{t \downarrow 0} \frac{1}{t} \nu_{t}(G \backslash N)=0
$$

for all Borel neighbourhoods $N$ of e. A measure $\nu \in \mathfrak{M}^{1}(G)$ is said to be a Gaussian measure if there exists a Gaussian semigroup $\left(\nu_{t}\right)_{t>0}$ in $\mathfrak{M}^{1}(G)$ with $\nu_{1}=\nu$.

The class of Gaussian measures in $\mathfrak{M}^{1}(G)$ will be abbreviated by $\mathcal{G}(G)$, and the subclass of its symmetric elements by $\mathcal{G}^{\mathrm{s}}(G)$.

If $\left(\nu_{t}\right)_{t>0}$ is a Gaussian semigroup in $\mathfrak{M}^{1}(G)$ then $\lim _{t \downarrow 0} \nu_{t}(G \backslash N)=0$ for all Borel neighbourhoods $N$ of $e$, hence $\nu_{0}(N)=1$ for all Borel neighbourhoods $N$ of $e$ with $\nu_{0}(\partial N)=0$. If $G$ is metrizable then this implies $\nu_{0}=\varepsilon_{e}$, thus $\left(\nu_{t}\right)_{t>0}$ is an $\{e\}$-continuous convolution semigroup.

The above definition slightly differs from the Definition 6.2.1 in Heyer [6], since here a Gaussian semigroup may consists of Dirac measures $\left(\varepsilon_{x_{t}}\right)_{t>0}$ belonging to a continuous one-parameter subsemigroup $\left(x_{t}\right)_{t \geqslant 0}$ of $G$.

It is known that for a Gaussian semigroup $\left(\nu_{t}\right)_{t>0}$ in $\mathfrak{M}^{1}(G)$ we have $\operatorname{supp}\left(\nu_{t}\right) \subset G_{0}$ for every $t>0$, where $G_{0}$ denotes the connected component of the identity $e$ (see Heyer [6, Theorem 6.2.3]). Moreover, if $G$ is a locally compact group and $G \neq\{e\}$ then $\mathcal{G}(G) \backslash \mathcal{D}(G) \neq \emptyset$, where $\mathcal{D}(G):=\left\{\varepsilon_{x}: x \in G\right\}$ (see Heyer [6, Theorem 6.2.8]). Consequently, if $G$ is not totally disconnected and $G \neq\{e\}$ then $\mathcal{G}(G) \backslash \mathcal{D}(G) \neq \emptyset$, and if $G$ is totally disconnected then $\mathcal{G}(G)=\left\{\varepsilon_{e}\right\}$.

2.3 Definition. A measure $\mu \in \mathfrak{M}^{1}(G)$ is called infinitely divisible if for all $n \in \mathbb{N}$ there exists $\mu_{\frac{1}{n}} \in \mathfrak{M}^{1}(G)$ such that $\mu=\left(\mu_{\frac{1}{n}}\right)^{n}$ (and hence $\mu_{\frac{1}{n}}$ can be considered as an $n$-th root of $\mu)$. The set of all infinitely divisible measures in $\mathfrak{M}^{1}(G)$ will be denoted by $\mathcal{I}(G)$.

2.4 Definition. A measure $\mu \in \mathfrak{M}^{1}(G)$ is called continuously embeddable if there exist a continuous convolution semigroup $\left(\mu_{t}\right)_{t>0}$ in $\mathfrak{M}^{1}(G)$ such that $\mu=\mu_{1}$. The set of all continuously embeddable measures in $\mathfrak{M}^{1}(G)$ will be denoted by $\mathcal{E}(G)$.

Clearly $\mathcal{G}^{\mathrm{s}}(G) \subset \mathcal{G}(G) \subset \mathcal{E}(G) \subset \mathcal{I}(G)$.

\section{Gaussian measures}

Let $G$ be a second countable locally compact Abelian group. The dual group of $G$ will be denoted by $\widehat{G}$. We will define Gaussian measures in the sense of Parthasarathy by their Fourier transforms.

3.1 Definition. The Fourier transform $\widehat{\mu}: \widehat{G} \rightarrow \mathbb{C}$ of a measure $\mu \in \mathfrak{M}^{1}(G)$ is defined by

$$
\widehat{\mu}(\chi):=\int_{G} \chi(x) \mu(\mathrm{d} x), \quad \chi \in \widehat{G} .
$$


The mapping $\mu \mapsto \widehat{\mu}$ from $\mathfrak{M}^{1}(G)$ into the set of mappings $\widehat{G} \rightarrow \mathbb{C}$ is injective.

3.2 Definition. A continuous function $\psi: \widehat{G} \rightarrow \mathbb{R}_{+}$is called a quadratic form on $\widehat{G}$ if

$$
\psi\left(\chi_{1} \chi_{2}\right)+\psi\left(\chi_{1} \chi_{2}^{-1}\right)=2\left(\psi\left(\chi_{1}\right)+\psi\left(\chi_{2}\right)\right) \quad \text { for all } \chi_{1}, \chi_{2} \in \widehat{G} .
$$

The set of all quadratic forms on $\widehat{G}$ will be denoted by $\mathbf{q}_{+}(\widehat{G})$.

3.3 Definition. A measure $\nu \in \mathfrak{M}^{1}(G)$ is said to be a Gaussian measure in the sense of Parthasarathy if there exist an element $m \in G$ and a quadratic form $\psi \in \mathrm{q}_{+}(\widehat{G})$ such that

$$
\widehat{\nu}(\chi)=\chi(m) \exp \{-\psi(\chi)\}
$$

holds for all $\chi \in \widehat{G}$.

The class of Gaussian measures in the sense of Parthasarathy in $\mathfrak{M}^{1}(G)$ will be abbreviated by $\mathcal{G}_{\mathrm{P}}(G)$, and the subclass of its symmetric elements by $\mathcal{G}_{\mathrm{P}}^{\mathrm{s}}(G)$.

3.4 Definition. A measure $\mu \in \mathfrak{M}^{1}(G)$ is called weakly infinitely divisible if for all $n \in \mathbb{N}$ there exist a measure $\mu_{n} \in \mathfrak{M}^{1}(G)$ and an element $x_{n} \in G$ such that $\mu=\mu_{n}^{n} * \varepsilon_{x_{n}}$. The collection of all weakly infinitely divisible measures in $\mathfrak{M}^{1}(G)$ will be denoted by $\mathcal{I}_{0}(G)$.

Obviously $\mathcal{I}(G) \subset \mathcal{I}_{0}(G)$.

3.5 Remark. For a bounded positive measure $\tau$ on $G$, the Poisson measure $\mathrm{e}^{\tau-\tau(G) \varepsilon_{e}} \in$ $\mathfrak{M}^{1}(G)$ with exponent $\tau$ is defined by

$$
\mathrm{e}^{\tau-\tau(G) \varepsilon_{e}}:=\mathrm{e}^{-\tau(G)}\left(\varepsilon_{e}+\tau+\frac{\tau * \tau}{2 !}+\frac{\tau * \tau * \tau}{3 !}+\cdots\right)
$$

A measure $\nu \in \mathfrak{M}^{1}(G)$ is a Gaussian measure in the sense of Parthasarathy if and only if it is weakly infinitely divisible and if for every factorisation of $\nu$ of the form $\nu=\mathrm{e}^{\tau-\tau(G) \varepsilon_{e}} * \lambda$ with a bounded positive measure $\tau$ on $G$ and a weakly infinitely divisible probability measure $\lambda$ one has $\tau=a \varepsilon_{e}$ for some $a \in \mathbb{R}_{+}$.

If $\nu \in \mathcal{G}_{\mathrm{P}}(G)$ then an element $m \in G$ and a quadratic form $\psi \in \mathrm{q}_{+}(\widehat{G})$ with the property such that $\widehat{\nu}(\chi)=\chi(m) \exp \{-\psi(\chi)\}$ holds for all $\chi \in \widehat{G}$ are uniquely determined.

Moreover, if $m \in G$ and $\psi \in \mathrm{q}_{+}(\widehat{G})$ then there exists $\nu \in \mathcal{G}_{\mathrm{P}}(G)$ such that $\widehat{\nu}(\chi)=\chi(m) \exp \{-\psi(\chi)\}$ holds for all $\chi \in \widehat{G}$.

(See Theorems 5.2.7 and 5.2.8 in Heyer [6].)

For $m \in G$ and $\psi \in \mathbf{q}_{+}(\widehat{G})$ let $\nu_{m, \psi} \in \mathfrak{M}^{1}(G)$ be defined by

$$
\widehat{\nu}_{m, \psi}(\chi)=\chi(m) \exp \{-\psi(\chi)\} \quad \text { for all } \chi \in \widehat{G} \text {. }
$$

Then

$$
\begin{aligned}
& \mathcal{G}_{\mathrm{P}}(G)=\left\{\nu_{m, \psi}: m \in G, \psi \in \mathrm{q}_{+}(\widehat{G})\right\} \\
& \mathcal{G}_{\mathrm{P}}^{\mathbf{s}}(G)=\left\{\nu_{m, \psi}: m \in G, m^{2}=e, \psi \in \mathbf{q}_{+}(\widehat{G})\right\}
\end{aligned}
$$


3.6 Definition. An element $x \in G$ is called continuously embeddable if there exists a continuous one-parameter subsemigroup $\left(x_{t}\right)_{t \geqslant 0}$ in $G$ such that $x_{1}=x$.

The set of continuously embeddable elements in $G$ will be denoted by $G_{\mathcal{E}}$.

Since $G$ is a locally compact Abelian group, $G_{\mathcal{E}}$ is a subgroup of $G$.

3.7 Theorem. Let $G$ be a second countable locally compact Abelian group. Then

$$
\mathcal{G}(G)=\left\{\nu_{m, \psi}: m \in G_{\mathcal{E}}, \psi \in \mathbf{q}_{+}(\widehat{G})\right\} .
$$

Consequently, $\mathcal{G}^{\mathrm{s}}(G) \subset \mathcal{G}_{\mathrm{P}}^{\mathrm{s}}(G) \cap \mathcal{G}(G)$ and $\subset \mathcal{G}_{\mathrm{P}}^{\mathrm{s}}(G) \cup \mathcal{G}(G) \subset \mathcal{G}_{\mathrm{P}}(G) \subset \mathcal{I}_{0}(G)$.

Proof. If $\nu \in \mathcal{G}(G)$ then there exists a Gaussian semigroup $\left(\nu_{t}\right)_{t>0}$ in $\mathfrak{M}^{1}(G)$ with $\nu_{1}=\nu$. Consequently, for all $t \geqslant 0$ we have that $\nu_{t}=\nu_{t / n}^{n}$ and $\lim _{n \rightarrow \infty} n \nu_{t / n}(G \backslash N)=0$ for all Borel neighbourhood $N$ of e. By Theorem 5.4.3 in Heyer [6], we obtain that $\nu_{t} \in \mathcal{G}_{\mathrm{P}}(G)$ for all $t \geqslant 0$. Thus there exist $m_{t} \in G$ and $\psi_{t} \in \mathrm{q}_{+}(\widehat{G})$ such that

$$
\widehat{\nu}_{t}(\chi)=\chi\left(m_{t}\right) \exp \left\{-\psi_{t}(\chi)\right\} \quad \text { for all } \chi \in \widehat{G} \text { and } t \geqslant 0 \text {. }
$$

The semigroup property $\nu_{s+t}=\nu_{s} * \nu_{t}$ for $s, t \geqslant 0$ implies $\widehat{\nu}_{s+t}(\chi)=\widehat{\nu}_{s}(\chi) \widehat{\nu}_{t}(\chi)$ for $\chi \in \widehat{G}$, thus

$$
\begin{aligned}
\widehat{\nu}_{s+t}(\chi) & =\chi\left(m_{s+t}\right) \exp \left\{-\psi_{s+t}(\chi)\right\}=\chi\left(m_{s}\right) \exp \left\{-\psi_{s}(\chi)\right\} \chi\left(m_{t}\right) \exp \left\{-\psi_{t}(\chi)\right\} \\
& =\chi\left(m_{s} m_{t}\right) \exp \left\{-\left(\psi_{s}(\chi)+\psi_{t}(\chi)\right)\right\} .
\end{aligned}
$$

Consequently $m_{s+t}=m_{s} m_{t}$ for all $s, t \geqslant 0$. Obviously $m_{0}=e$. Moreover

$$
t \mapsto \chi\left(m_{t}\right)=\frac{\widehat{\nu}_{t}(\chi)}{\left|\widehat{\nu}_{t}(\chi)\right|}
$$

is a continuous mapping from $\mathbb{R}_{+}$into $\mathbb{C}$ for all $\chi \in \widehat{G}$, hence $\left(m_{t}\right)_{t \geqslant 0}$ is a continuous one-parameter subsemigroup in $G$. Consequently $\nu=\nu_{m_{1}, \psi_{1}}$ and $m_{1} \in G_{\mathcal{E}}$.

Suppose now that $\nu=\nu_{m, \psi}$ with $m \in G_{\mathcal{E}}$ and $\psi \in \mathrm{q}_{+}(\widehat{G})$. Then there exist a continuous one-parameter subsemigroup $\left(m_{t}\right)_{t \geqslant 0}$ in $G$ such that $m_{1}=m$. For all $t \geqslant 0$ there exists a measure $\nu_{t} \in \mathcal{G}_{\mathrm{P}}(G)$ such that

$$
\widehat{\nu}_{t}(\chi)=\chi\left(m_{t}\right) \exp \{-t \psi(\chi)\} \quad \text { for all } \chi \in \widehat{G} \text {. }
$$

Clearly $\left(\nu_{t}\right)_{t>0}$ is an $\{e\}$-continuous convolution semigroup in $\mathfrak{M}^{1}(G)$ such that $\nu_{1}=\nu$. Consider the related continuous convolution hemigroup $\mu_{s, t}:=\nu_{t-s}, 0 \leqslant s \leqslant t$. Let $\left(X_{t}\right)_{t \geqslant 0}$ be an associated (time-homogeneous) increment process in $G$. By Theorem 2.6 in Bingham and Heyer [4] we conclude that $\left(X_{t}\right)_{t \geqslant 0}$ has a. s. continuous paths. By Corollary 2 of Theorem 2 in Siebert [15] we obtain that $\left(\mu_{s, t}\right)_{0 \leqslant s \leqslant t}$ is a diffusion hemigroup in the sense that

$$
\lim _{Z \in \mathfrak{Z}_{[0, T]}} \sum_{k=1}^{n} \mu_{\tau_{k-1}, \tau_{k}}(G \backslash N)=0
$$

for all Borel neighbourhood $N$ of $e$ and for all $T>0$. This implies that $\left(\nu_{t}\right)_{t>0}$ is a Gaussian semigroup. Indeed, for all $\varepsilon>0$ there exists $\delta>0$ such that $\sum_{k=1}^{n} \mu_{\tau_{k-1}, \tau_{k}}(G \backslash N) \leqslant \varepsilon$ for all decomposition $Z:=\left\{0=\tau_{0}<\tau_{1}<\cdots<\tau_{n}=1\right\}$ of $[0,1]$ with $|Z|<\delta$. Hence $n \nu_{t}(G \backslash N) \leqslant \varepsilon$ if $t<\delta$ and $n t \leqslant 1$, which clearly implies that $\lim _{t \rightarrow 0} \frac{1}{t} \nu_{t}(G \backslash N)=0$. Consequently $\nu \in \mathcal{G}(G)$. 


\section{Continuously embeddable elements}

Let again $G$ be a second countable locally compact Abelian group. First observe that an element of $G$ is continuously embeddable if and only if the related Dirac measure is a continuously embeddable measure, i.e., $x \in G_{\mathcal{E}}$ if and only if $\varepsilon_{x} \in \mathcal{E}(G)$. Consequently, $G_{\mathcal{E}}=\left\{x \in G: \varepsilon_{x} \in \mathcal{E}(G)\right\}$ and $\left\{\varepsilon_{x}: x \in G_{\mathcal{E}}\right\} \subset \mathcal{G}(G)$. Moreover, by Theorem 3.7, $\mathcal{G}(G)=\mathcal{G}_{\mathrm{P}}(G)$ holds if and only if $\left\{\varepsilon_{x}: x \in G\right\} \subset \mathcal{E}(G)$.

Further, note that each continuous one-parameter subsemigroup $\left(x_{t}\right)_{t \geqslant 0}$ of $G$ can be extended to a continuous one-parameter subgroup $\left(x_{t}\right)_{t \in \mathbb{R}}$ of $G$ by

$$
x_{t}:=\left(x_{-t}\right)^{-1}, \quad t<0 .
$$

Hence

$$
G_{\mathcal{E}}=\bigcup\{\varphi(\mathbb{R}): \varphi \in \operatorname{Hom}(\mathbb{R}, G)\} \subset G_{0},
$$

where $\operatorname{Hom}(\mathbb{R}, G)$ denotes the set of continuous homomorphisms from the additive group $\mathbb{R}$ into $G$. Consequently, if $G$ is not connected then $G_{\mathcal{E}} \neq G$, and hence $\mathcal{G}(G) \neq \mathcal{G}_{\mathrm{P}}(G)$.

Moreover, $G_{\mathcal{E}}$ is a dense subgroup of $G_{0}$, and $G_{\mathcal{E}}$ equals to the union of the arcs of $G$ which contain $e$, i.e., $G_{\mathcal{E}}$ is the arc-component of $e$. Combining Theorem 3.7 with the results of Dixmier [5] we conclude the following theorem.

4.1 Theorem. Let $G$ be a second countable locally compact Abelian group. Then the following statements are equivalent:

(i) $\mathcal{G}(G)=\mathcal{G}_{\mathrm{P}}(G)$;

(ii) $G_{\mathcal{E}}=G$;

(iii) $\left\{\varepsilon_{x}: x \in G\right\} \subset \mathcal{E}(G)$;

(iv) $G$ is the union of all continuous one-parameter subgroups of $G$;

(v) $G$ is arcwise connected;

(vi) $G=\mathbb{R}^{n} \times \mathbb{T}^{I}$, where $n \in \mathbb{Z}_{+}$and $I$ is an at most countable set.

Note that each solenoidal group $\Sigma_{c}$ is a compact connected Abelian group with $\left(\Sigma_{c}\right)_{\mathcal{E}} \neq$ $\Sigma_{c}$ (see Example 5.4).

\section{$5 \quad$ Examples}

\subsection{Gaussian measures on $\mathbb{R}^{d}$.}

Consider the group $G=\mathbb{R}^{d}$, where $d \in \mathbb{N}$. The character group $\left(\mathbb{R}^{d}\right)^{\wedge}$ is topologically isomorphic with $\mathbb{R}^{d}$. Every continuous character of $\mathbb{R}^{d}$ has the form $\chi_{y}(x)=\mathrm{e}^{i\langle x, y\rangle}$, $x \in \mathbb{R}^{d}$ for some $y \in \mathbb{R}^{d}$. Every quadratic form $\psi$ on $\left(\mathbb{R}^{d}\right)^{\wedge} \cong \mathbb{R}^{d}$ has the form 
$\psi_{B}\left(\chi_{y}\right)=\langle B y, y\rangle$ for some symmetric positive semidefinite matrix $B \in \mathbb{R}^{d \times d}$. Clearly $\left(\mathbb{R}^{d}\right)_{\mathcal{E}}=\mathbb{R}^{d}$, hence

$$
\begin{aligned}
\mathcal{G}\left(\mathbb{R}^{d}\right) & =\mathcal{G}_{\mathrm{P}}\left(\mathbb{R}^{d}\right)=\left\{\nu_{a, B}: a \in \mathbb{R}^{d}, B \in \mathbb{R}^{d \times d} \text { with } B^{\top}=B \text { and } B \geqslant 0\right\}, \\
\mathcal{G}^{\mathrm{s}}\left(\mathbb{R}^{d}\right) & =\mathcal{G}_{\mathrm{P}}^{\mathrm{s}}\left(\mathbb{R}^{d}\right)=\left\{\nu_{0, B}: B \in \mathbb{R}^{d \times d} \text { with } B^{\top}=B \text { and } B \geqslant 0\right\},
\end{aligned}
$$

where $\nu_{a, B} \in \mathfrak{M}^{1}\left(\mathbb{R}^{d}\right)$ is defined by

$$
\widehat{\nu}_{a, B}\left(\chi_{y}\right):=\exp \left\{i\langle a, y\rangle-\frac{1}{2}\langle B y, y\rangle\right\}, \quad \text { for all } y \in \mathbb{R}^{d} \cong\left(\mathbb{R}^{d}\right)^{\wedge}
$$

\subsection{Gaussian measures on $\mathbb{T}^{d}$.}

Consider the group $G=\mathbb{T}^{d}$, where $\mathbb{T}:=\left\{\mathrm{e}^{2 \pi i x}: 0 \leqslant x<1\right\}$ denotes the torus group. The character group $\left(\mathbb{T}^{d}\right)^{\wedge}$ is topologically isomorphic with $\mathbb{Z}^{d}$. Every continuous character of $\mathbb{T}^{d}$ has the form $\chi_{m}(x)=\prod_{k=1}^{d} x_{k}^{m_{k}}, x=\left(x_{1}, \ldots, x_{d}\right) \in \mathbb{T}^{d}$ for some $m=\left(m_{1}, \ldots, m_{d}\right) \in$ $\mathbb{Z}^{d}$. Every quadratic form $\psi$ on $\left(\mathbb{T}^{d}\right)^{\wedge} \cong \mathbb{Z}^{d}$ has the form $\psi_{B}\left(\chi_{m}\right)=\langle B m, m\rangle$ for some symmetric positive semidefinite matrix $B \in \mathbb{R}^{d \times d}$. Clearly $\left(\mathbb{T}^{d}\right)_{\mathcal{E}}=\mathbb{T}^{d}$, hence

$$
\begin{aligned}
\mathcal{G}\left(\mathbb{T}^{d}\right) & =\mathcal{G}_{\mathrm{P}}\left(\mathbb{T}^{d}\right)=\left\{\nu_{a, B}: a \in \mathbb{T}^{d}, \quad B \in \mathbb{R}^{d \times d} \text { with } B^{\top}=B, \quad B \geqslant 0\right\}, \\
\mathcal{G}^{\mathrm{s}}\left(\mathbb{T}^{d}\right) & =\mathcal{G}_{\mathrm{P}}^{\mathbf{s}}\left(\mathbb{T}^{d}\right)=\left\{\nu_{a, B}: a \in \mathbb{T}^{d} \text { with } a_{i} \in\{1,-1\}, \quad B \in \mathbb{R}^{d \times d} \text { with } B^{\top}=B, \quad B \geqslant 0\right\},
\end{aligned}
$$

where $\nu_{a, B} \in \mathfrak{M}^{1}\left(\mathbb{T}^{d}\right)$ is defined by

$$
\widehat{\nu}_{a, B}\left(\chi_{m}\right):=\left(\prod_{k=1}^{d} a_{k}^{m_{k}}\right) \exp \left\{-\frac{1}{2}\langle B m, m\rangle\right\}, \quad \text { for all } m=\left(m_{1}, \ldots, m_{d}\right) \in \mathbb{Z}^{d} \cong\left(\mathbb{T}^{d}\right)^{\wedge}
$$

Writing the element $a=\left(a_{1}, \ldots, a_{d}\right) \in \mathbb{T}^{d}$ in the form $a=\left(\mathrm{e}^{2 \pi i \alpha_{1}}, \ldots, \mathrm{e}^{2 \pi i \alpha_{d}}\right)$ with $0 \leqslant \alpha_{k}<1, \quad k=1, \ldots, d$, we obtain $\chi_{m}(a)=\mathrm{e}^{2 \pi i\langle\alpha, m\rangle}$ with $\alpha:=\left(\alpha_{1}, \ldots, \alpha_{d}\right)$, and

$$
\widehat{\nu}_{a, B}\left(\chi_{m}\right)=\exp \left\{2 \pi i\langle\alpha, m\rangle-\frac{1}{2}\langle B m, m\rangle\right\}, \quad \text { for all } m=\left(m_{1}, \ldots, m_{d}\right) \in \mathbb{Z}^{d} \cong\left(\mathbb{T}^{d}\right)^{\wedge} .
$$

\subsection{Gaussian measures on $\mathbb{T}^{\infty}$.}

Consider the group $\mathbb{T}^{\infty}:=\prod_{k=1}^{\infty} G_{k}$, where $G_{k}:=\mathbb{T}$ for all $k \in \mathbb{N}$. The character group $\left(\mathbb{T}^{\infty}\right)^{\wedge}$ is topologically isomorphic with $\mathbb{Z}^{(\infty)}$ consisting of the elements of $\mathbb{Z}^{\infty}$ having only finitely many nonzero coordinates. Every continuous character of $\mathbb{T}^{\infty}$ has the form $\chi_{m}(x)=\prod_{k=1}^{\infty} x_{k}^{m_{k}}, \quad x=\left(x_{1}, x_{2}, \ldots\right) \in \mathbb{T}^{\infty}$ for some $m=\left(m_{1}, m_{2}, \ldots\right) \in \mathbb{Z}^{(\infty)}$. Every quadratic form $\psi$ on $\left(\mathbb{T}^{\infty}\right)^{\wedge} \cong \mathbb{Z}^{(\infty)}$ has the form $\psi_{B}\left(\chi_{m}\right)=\sum_{j, k=1}^{\infty} b_{j, k} m_{j} m_{k}$ for some symmetric positive semidefinite matrix $B=\left(b_{j, k}\right)_{j, k=1,2, \ldots} \in \mathbb{R}^{\infty \times \infty}$ (i.e., $B_{d}:=$ $\left(b_{j, k}\right)_{j, k=1, \ldots, d} \in \mathbb{R}^{d \times d}$ is positive semidefinite for all $\left.d \in \mathbb{N}\right)$. Clearly $\left(\mathbb{T}^{\infty}\right)_{\mathcal{E}}=\mathbb{T}^{\infty}$, hence

$$
\begin{aligned}
\mathcal{G}\left(\mathbb{T}^{\infty}\right) & =\mathcal{G}_{\mathrm{P}}\left(\mathbb{T}^{\infty}\right)=\left\{\nu_{a, B}: a \in \mathbb{T}^{\infty}, \quad B \in \mathbb{R}^{\infty \times \infty} \text { with } B^{\top}=B, \quad B \geqslant 0\right\}, \\
\mathcal{G}^{\mathrm{s}}\left(\mathbb{T}^{\infty}\right) & =\mathcal{G}_{\mathrm{P}}^{\mathrm{s}}\left(\mathbb{T}^{\infty}\right)=\left\{\nu_{a, B}: a \in \mathbb{T}^{\infty} \text { with } a_{i} \in\{1,-1\}, \quad B \in \mathbb{R}^{\infty \times \infty} \text { with } B^{\top}=B, \quad B \geqslant 0\right\},
\end{aligned}
$$


where $\nu_{a, B} \in \mathfrak{M}^{1}\left(\mathbb{T}^{\infty}\right)$ is defined by

$$
\widehat{\nu}_{a, B}\left(\chi_{m}\right):=\left(\prod_{k=1}^{\infty} a_{k}^{m_{k}}\right) \exp \left\{-\frac{1}{2} \sum_{j, k=1}^{\infty} b_{j, k} m_{j} m_{k}\right\}
$$

for all $m=\left(m_{1}, m_{2}, \ldots\right) \in \mathbb{Z}^{(\infty)} \cong\left(\mathbb{T}^{\infty}\right)^{\wedge}$. Writing the element $a=\left(a_{1}, a_{2}, \ldots\right) \in \mathbb{T}^{\infty}$ in the form $a=\left(\mathrm{e}^{2 \pi i \alpha_{1}}, \mathrm{e}^{2 \pi i \alpha_{2}}, \ldots\right)$ with $0 \leqslant \alpha_{k}<1, k=1,2, \ldots$, we obtain $\chi_{m}(a)=$ $\exp \left\{2 \pi i \sum_{k=1}^{\infty} \alpha_{k} m_{k}\right\}$, and

$$
\widehat{\nu}_{a, B}\left(\chi_{m}\right)=\exp \left\{2 \pi i \sum_{k=1}^{\infty} \alpha_{k} m_{k}-\frac{1}{2} \sum_{j, k=1}^{\infty} b_{j, k} m_{j} m_{k}\right\}
$$

for all $m=\left(m_{1}, m_{2}, \ldots\right) \in \mathbb{Z}^{(\infty)} \cong\left(\mathbb{T}^{\infty}\right)^{\wedge}$.

\subsection{Gaussian measures on solenoidal groups.}

Let $c=\left(c_{1}, c_{2}, \ldots\right) \in \mathbb{N}^{\infty}$ with $c_{k} \geqslant 2$ for $k \in \mathbb{N}$. The solenoid $\Sigma_{c}$ can be considered as a subgroup of $\mathbb{T}^{\infty}$, namely,

$$
\Sigma_{c}:=\left\{x=\left(x_{1}, x_{2}, \ldots\right) \in \mathbb{T}^{\infty}: x_{k}=x_{k+1}^{c_{k}} \text { for all } k \in \mathbb{N}\right\} .
$$

Thus for $x=\left(x_{1}, x_{2}, \ldots\right) \in \Sigma_{c}$ we have $x_{j}=x_{k}^{c_{j} c_{j+1} \ldots c_{k-1}}=x_{k}^{c_{[j, k)}}$ for $j \leqslant k$, where $c_{[j, k)}:=c_{j} c_{j+1} \ldots c_{k-1}$ for $j<k$ and $c_{[k, k)}:=1$. In fact, $\Sigma_{c}$ is the projective limit of the projective sequence $\left(G_{k}, \pi_{j, k}\right), 1 \leqslant j \leqslant k$, where $G_{k}:=\mathbb{T}$ for all $k \in \mathbb{N}$, and $\pi_{j, k}: G_{k} \rightarrow G_{j}$ is the mapping $z \mapsto z^{c[j, k)}$. It follows that $\Sigma_{c}$ is a second countable compact connected group. It is well known that $\Sigma_{c}$ is not locally connected and not arcwise connected. The arc-component of the identity $e=(1,1, \ldots)$ of $\Sigma_{c}$ (i.e., the subgroup of continuously embeddable elements of $\Sigma_{c}$ ) is

$$
\left(\Sigma_{c}\right)_{\mathcal{E}}=\left\{\left(\mathrm{e}^{2 \pi i \lambda}, \mathrm{e}^{2 \pi i \lambda / c_{1}}, \mathrm{e}^{2 \pi i \lambda /\left(c_{1} c_{2}\right)}, \ldots\right): \lambda \in \mathbb{R}\right\} .
$$

If $m=\left(m_{1}, m_{2}, \ldots\right) \in \mathbb{Z}^{(\infty)} \cong\left(\mathbb{T}^{\infty}\right)^{\wedge}$ then there exists $k \in \mathbb{N}$ such that $m_{j}=0$ for all $j>k$, hence

$$
\chi_{m}(x)=\prod_{j=1}^{k} x_{j}^{m_{j}}=\prod_{j=1}^{k} x_{k}^{m_{j} c_{[j, k)}}=x_{k}^{m_{1} c_{[1, k)}+\cdots+m_{k-1} c_{[k-1, k)}+m_{k}} .
$$

Consequently, the character group $\widehat{\Sigma}_{c}$ is topologically isomorphic with the (discrete) additive group

$$
\left\{\gamma=\frac{\ell}{c_{[1, k)}}: \ell \in \mathbb{Z}, k \in \mathbb{N}\right\}
$$

of rational numbers. Every continuous character of $\Sigma_{c}$ has the form $\chi_{\gamma}(x)=x_{k}^{\ell}, \quad x=$ $\left(x_{1}, x_{2}, \ldots\right) \in \Sigma_{a}$ for some $\gamma=\frac{\ell}{c_{[1, k)}}, \ell \in \mathbb{Z}, k \in \mathbb{N}$. Every quadratic form $\psi$ on $\widehat{\Sigma}_{c}$ has the form $\psi_{b}(\gamma)=b \gamma^{2}$ for some $b \geqslant 0$. Hence

$$
\begin{aligned}
\mathcal{G}\left(\Sigma_{c}\right) & =\left\{\nu_{a, b}: a \in\left(\Sigma_{c}\right)_{\mathcal{E}}, b \geqslant 0\right\}, \\
\mathcal{G}_{\mathrm{P}}\left(\Sigma_{c}\right) & =\left\{\nu_{a, b}: a \in \Sigma_{c}, b \geqslant 0\right\}, \\
\mathcal{G}^{\mathrm{s}}\left(\Sigma_{c}\right)=\mathcal{G}_{\mathrm{P}}^{\mathrm{s}}\left(\Sigma_{c}\right) & =\left\{\nu_{a, b}: a \in \Sigma_{c} \text { with } a_{i} \in\{1,-1\}, b \geqslant 0\right\},
\end{aligned}
$$


where $\nu_{a, b} \in \mathfrak{M}^{1}\left(\Sigma_{c}\right)$ is defined by

$$
\widehat{\nu}_{a, b}\left(\chi_{\gamma}\right):=a_{k}^{\ell} \exp \left\{-\frac{1}{2} b \gamma^{2}\right\}, \quad \text { for all } \gamma=\frac{\ell}{c_{[1, k)}} \in \widehat{\Sigma}_{c} .
$$

Note that there exists an element $a \in \Sigma_{c}$ with $a_{i} \in\{1,-1\}$ and $a \neq e$ if and only if there are only at most finitely many even numbers among $\left\{c_{k}: k \in \mathbb{N}\right\}$. Moreover, if $a \in\left(\Sigma_{c}\right)_{\mathcal{E}}$ with $a_{i} \in\{1,-1\}$ then $a=e$.

If $a \in\left(\Sigma_{c}\right)_{\mathcal{E}}$ then there exists $\lambda \in \mathbb{R}$ such that $a=\left(\mathrm{e}^{2 \pi i \lambda}, \mathrm{e}^{2 \pi i \lambda / c_{1}}, \mathrm{e}^{2 \pi i \lambda /\left(c_{1} c_{2}\right)}, \ldots\right)$, hence for $\gamma=\frac{\ell}{c_{[1, k)}} \in \widehat{\Sigma}_{c}$ we have $\chi_{\gamma}(a)=a_{k}^{\ell}=\mathrm{e}^{2 \pi i \lambda \ell / c_{[1, k)}}=\mathrm{e}^{2 \pi i \lambda \gamma}$. Consequently,

$$
\widehat{\nu}_{a, b}\left(\chi_{\gamma}\right)=\exp \left\{2 \pi i \lambda \gamma-\frac{1}{2} b \gamma^{2}\right\}, \quad \text { for all } \gamma \in \widehat{\Sigma}_{c} .
$$

Now let $a=\left(a_{1}, a_{2}, \ldots\right) \in \Sigma$. Write $a_{k} \in \mathbb{T}$ in the form $a_{k}=\mathrm{e}^{2 \pi i \alpha_{k}}$ with $0 \leqslant \alpha_{k}<1$. Since $a_{1}=a_{2}^{c_{1}}$, there exists an integer $j_{1} \in \mathbb{Z}$ such that $\alpha_{1}=c_{1} \alpha_{2}-j_{1}$, hence $a_{2}=\mathrm{e}^{2 \pi i\left(\alpha_{1}+j_{1}\right) / c_{1}}$. In a similar way one obtains $a_{k}=\mathrm{e}^{2 \pi i\left(\alpha_{1}+j_{1}+\cdots+c_{[1, k-1)} j_{k-1}\right) / c_{[1, k)}}$ for all $k \in \mathbb{N}$ with some $j=\left(j_{1}, j_{2}, \ldots\right) \in \mathbb{Z}^{\infty}$. Hence for $\gamma=\frac{\ell}{c_{[1, k)}} \in \widehat{\Sigma}_{c}$ we have $\chi_{\gamma}(a)=a_{k}^{\ell}=\mathrm{e}^{2 \pi i\left(\alpha_{1}+j_{1}+\cdots+c_{[1, k-1)} j_{k-1}\right) \ell / c_{[1, k)}}=\mathrm{e}^{2 \pi i\left(\alpha_{1}+j_{1}+\cdots+c_{[1, k-1)} j_{k-1}\right) \gamma}$. Consequently,

$$
\widehat{\nu}_{a, b}\left(\chi_{\gamma}\right)=\exp \left\{2 \pi i\left(\alpha_{1}+j_{1}+\cdots+c_{[1, k-1)} j_{k-1}\right) \gamma-\frac{1}{2} b \gamma^{2}\right\}, \quad \text { for all } \gamma \in \widehat{\Sigma}_{c} .
$$

Note that each Gaussian measure on $\Sigma_{c}$ in the sense of Parthasarathy has full support and is singular with respect to a Haar measure on $\Sigma_{c}$. Moreover, two Gaussian measures $\nu_{a, b}, \nu_{a^{\prime}, b^{\prime}} \in \mathcal{G}_{\mathrm{P}}\left(\Sigma_{c}\right)$ in the sense of Parthasarathy are mutually absolutely continuous if $a^{-1} a^{\prime} \in(\Sigma)_{\mathcal{E}}$, otherwise they are singular with respect to each other. Particularly, any two Gaussian measures $\nu_{a, b}, \nu_{a^{\prime}, b^{\prime}} \in \mathcal{G}\left(\Sigma_{c}\right)$ are mutually absolutely continuous. (See Bendikov and Saloff-Coste $[2,5.2]$.)

\section{$6 \quad$ Weakly infinitely divisible measures}

The purpose of this section is to characterize the set of locally compact Abelian groups $G$ for which each weakly infinitely divisible measure is embeddable, i.e., $\mathcal{I}_{0}(G)=\mathcal{E}(G)$.

6.1 Theorem. Let $G$ be a second countable locally compact Abelian group. Then the following statements are equivalent:

(i) $G$ is arcwise connected;

(ii) $\mathcal{I}_{0}(G)=\mathcal{I}(G)=\mathcal{E}(G)$.

Proof. (i) $\Longrightarrow$ (ii). Let us consider an arbitrary measure $\mu \in \mathcal{I}_{0}(G)$. We are going to show that $\mu \in \mathcal{E}(G)$. Let $g: G \times \widehat{G} \rightarrow \mathbb{R}$ be a local inner product (see Definition 5.1.7 in Heyer [6]). Then by Corollary IV.7.1 in Parthasarathy [13], the Fourier transform $\widehat{\mu}$ admits a representation

$$
\widehat{\mu}(\chi)=\widehat{\omega}_{H}(\chi) \chi(m) \exp \left\{-\psi(\chi)+\int_{G}(\chi(x)-1-i g(x, \chi)) \eta(\mathrm{d} x)\right\}
$$


for all $\chi \in \widehat{G}$, where $H$ is a compact subgroup of $G, m \in G, \psi \in \mathrm{q}_{+}(\widehat{G})$ and $\eta$ is a Lévy measure on $G$, i.e., $\eta$ is a positive Borel measure on $G$ such that $\eta(\{e\})=0$, $\eta(G \backslash N)<\infty$ for all Borel neighbourhood $N$ of $e$, and $\int_{G}(1-\operatorname{Re} \chi(x)) \eta(\mathrm{d} x)<\infty$ for all $\chi \in \widehat{G}$. Since $G$ is arcwise connected, there exists a continuous one-parameter subsemigroup $\left(m_{t}\right)_{t \geqslant 0}$ in $G$ such that $m_{1}=m$ (see Theorem 4.1). Clearly $t \psi \in \mathrm{q}_{+}(\widehat{G})$ and $t \eta$ is a Lévy measure on $G$ for all $t \geqslant 0$. By Theorem IV.7.1 in Parthasarathy [13], for all $t \geqslant 0$ there exists a measure $\mu_{t} \in \mathcal{I}(G)$ such that

$$
\widehat{\mu}_{t}(\chi)=\widehat{\omega}_{H}(\chi) \chi\left(m_{t}\right) \exp \left\{-t \psi(\chi)+t \int_{G}(\chi(x)-1-i g(x, \chi)) \eta(\mathrm{d} x)\right\}
$$

for all $\chi \in \widehat{G}$. We have

$$
\widehat{\omega}_{H}(\chi)= \begin{cases}1 & \text { if } \chi(x)=1 \text { for all } x \in H, \\ 0 & \text { otherwise. }\end{cases}
$$

(In fact, the set $\{\chi \in \widehat{G}: \chi(x)=1$ for all $x \in H\}$ is the annihilator of $H$ in $\widehat{G}$.) Hence we have $\widehat{\omega}_{H}(\chi)^{2}=\widehat{\omega}_{H}(\chi)$ for all $\chi \in \widehat{G}$. Moreover, for each $s, t>0$ and $\chi \in \widehat{G}$, we have $\chi\left(m_{s}\right) \chi\left(m_{t}\right)=\chi\left(m_{s} m_{t}\right)=\chi\left(m_{s+t}\right)$, thus we can conclude $\widehat{\mu}_{s}(\chi) \widehat{\mu}_{t}(\chi)=\widehat{\mu}_{s+t}(\chi)$. Further, $\lim _{t \downarrow 0} \widehat{\mu}_{t}(\chi)=\widehat{\omega}_{H}(\chi)$ for all $\chi \in \widehat{G}$ implies $\lim _{t \downarrow 0} \mu_{t}=\omega_{H}$. Consequently $\left(\mu_{t}\right)_{t>0}$ is a $H$-continuous convolution semigroup in $\mathfrak{M}^{1}(G)$ with $\mu_{1}=\mu$, and we obtain $\mu \in \mathcal{E}(G)$.

(ii) $\Longrightarrow$ (i). If $G$ is not arcwise connected then consider an element $x \in G$ such that $x \notin G_{\mathcal{E}}$ (see Theorem 4.1). Then $\varepsilon_{x} \in \mathcal{I}_{0}(G)$ but $\varepsilon_{x} \notin \mathcal{E}(G)$, hence $\mathcal{I}_{0}(G) \neq \mathcal{E}(G)$.

\section{$7 \quad$ Embedding property}

A locally compact group $G$ is said to satisfy the embedding property if $\mathcal{I}(G)=\mathcal{E}(G)$. If $G$ is a locally compact Abelian group such that any $\mu \in \mathcal{I}(G)$ is root compact (i.e., the root set $R(\mu):=\cup_{n \in \mathbb{N}}\left\{\nu^{m}: \nu \in \mathfrak{M}^{1}(G)\right.$ with $\left.\nu^{n}=\nu, 1 \leqslant m \leqslant n\right\}$ is relatively compact in $\mathfrak{M}^{1}(G)$ ) then by Theorem 3.5.12 in Heyer [6] the following statements are equivalent:

(i) $G_{0}$ is locally arcwise connected;

(ii) $\mathcal{I}(G)=\mathcal{E}(G)$.

We note that by Theorem 3.5.12 in Heyer [6] for any locally compact Abelian group $G$ the following statements are equivalent:

(i) any $\mu \in \mathcal{I}(G)$ is root compact;

(ii) the set of all compact elements of $G$ is compact, and the set of divisible elements of $G$ equals $G_{0}$.

In view of the above results one might conjecture that a locally compact Abelian group satisfies the embedding property if and only if any $\mu \in \mathcal{I}(G)$ is root compact and $G_{0}$ is locally arcwise connected, but such a result is false, as it is shown in Heyer [6, 3.5.21]. 
We can give a sufficient condition for a locally compact Abelian group in order to satisfy the embedding property in the following way. One can extend the statement of Theorem 4.1 along Dixmier [5] (see Heyer [6, Theorem B]). From this source follows that the class of second countable locally compact Abelian groups $G$ with arcwise connected dual $\widehat{G}$ consists of Abelian aperiodic groups which can be represented as closed subgroups of a locally convex vector space, that is as products

$$
\mathbb{R}^{n} \times \mathbb{Z}^{I}
$$

with a countable set $I$ (of cardinality $|I|$ ), embeddable into

$$
\mathbb{R}^{n+|I|} \text {. }
$$

(Note that a second countable locally compact Abelian group $G$ is aperiodic if and only if $\widehat{G}=\mathbb{R}^{n} \times K$ with $n \geqslant 0$ and with a connected and compact $K$ such that $\widehat{K}$ is torsion-free, see Heyer [6, 3.5.15, 3.5.18].) From Siebert's paper [14, 6, Satz 4] follows that this closed subgroup of the vector space is strongly root compact. In fact, if $|I|$ is infinite, then there exists a closed, discrete, free subgroup $N$ of $\mathbb{Z}^{I}$ which is uniformly root compact such that

$$
G=N \times \mathbb{R}^{n}
$$

Now, apply Theorem 3.1.11 in Heyer [6], and one gets the uniform root compactness of $G$. From this it follows by Theorem 3.5.8 (or Corollary 3.5.9) of Heyer [6] that for a second countable locally compact Abelian group $G$ with arcwise connected dual $\widehat{G}$ we have $\mathcal{I}(G)=\mathcal{E}(G)$, and for each $\mu \in \mathcal{I}(G)$ there exists a unique $\{e\}$-continuous convolution semigroup $\left(\mu_{t}\right)_{t>0}$ with $\mu_{1}=\mu$.

The aim of this section is to give another proof of the above statement.

7.1 Lemma. (Existence and uniqueness of the logarithm)

(i) Let $S$ be a locally arcwise connected topological space, and let $\left(f_{n}\right)_{n \geqslant 1}$ be a sequence of continuous mappings $f_{n}: S \rightarrow \mathbb{C} \backslash\{0\}$ satisfying $f_{n+1}^{2}=f_{n}$ for all $n \geqslant 1$. Then there exists a continuous function $h: S \rightarrow \mathbb{C}$ with $f_{1}=\exp h$.

(ii) Let $S$ be a connected topological space, $a \in S$ and $f: S \rightarrow \mathbb{C} \backslash\{0\}$ a continuous mapping with $f(a)=1$. Then there exists at most one continuous function $h: S \rightarrow \mathbb{C}$ satisfying $f=\exp h$ and $h(a)=0$.

Proof. (i). Since every connected component of $S$ is open, we may assume without loss of generality that $S$ is connected. We fix $a \in S$ and assume that $f_{n}(a)=1$ for all $n \geqslant 1$. Let

$$
H:=\{z \in \mathbb{C}: \operatorname{Re} z>0\}
$$

and let $U_{n}$ denote the connected component of

$$
\left\{s \in S: f_{n}(s) \in H\right\}
$$

which contains $a$. Then $\left(U_{n}\right)_{n \geqslant 1}$ is an increasing sequence of connected open subsets of $S$. For every continuous arc $\gamma:[0,1] \rightarrow S$ with $\gamma(0)=a$ and sufficiently large $n$ we have 
$\gamma([0,1]) \subset U_{n}$ or $S=\bigcup_{n \geqslant 1} U_{n}$. Indeed, there exists a continuous function $\varphi:[0,1] \rightarrow \mathbb{C}$ with $f_{1} \circ \gamma=\exp \varphi$. But then, for large $n$ and $\psi:=2^{1-n} \varphi$ the function $f_{n} \circ \gamma=\exp \psi$ takes values in $H$.

Now let $\log$ denote the principle branch of the logarithm on $H$ and define $h_{n}$ on $U_{n}$ by

$$
h_{n}(s):=2^{n-1} \log f_{n}(s)
$$

for each $n \geqslant 1$. The function $h_{n}$ is continuous on $U_{n}$ and coincides with $h_{n+1}$ on $U_{n}$. Thus there exists a function $h: S \rightarrow \mathbb{C}$ satisfying $\operatorname{Res}_{U_{n}} h=h_{n}$ for all $n \geqslant 1$. The function $h$ is the desired logarithm.

(ii). Let $h$ and $h^{\prime}$ be two continuous functions $S \rightarrow \mathbb{C}$ satisfying

$$
f=\exp h=\exp h^{\prime}
$$

and $h(a)=h^{\prime}(a)=0$. Then the continuous function $h^{\prime}-h$ takes on only values in $2 \pi i \mathbb{Z}$ and is therefore constant. Thus $h^{\prime}=h$, since $h^{\prime}(a)-h(a)=0$.

7.2 Corollary. Let $S$ be a connected and locally arcwise connected topological space, and let $a \in S$. Moreover, let $f: S \rightarrow \mathbb{C} \backslash\{0\}$ be a continuous mapping with $f(a)=1$. We assume that for every $n \geqslant 1$ there exists a continuous mapping $g_{n}: S \rightarrow \mathbb{C} \backslash\{0\}$ such that $g_{n}^{n}=f$. Then there exists exactly one continuous function $h$ on $S$ with $h(a)=0$ satisfying

$$
f=\exp h
$$

7.3 Theorem. Let $G$ be a second countable locally compact Abelian group with arcwise connected dual $\widehat{G}$. Then

$$
\mathcal{I}(G)=\mathcal{E}(G) .
$$

Moreover, for each $\mu \in \mathcal{I}(G)$ there exists a unique $\{e\}$-continuous convolution semigroup $\left(\mu_{t}\right)_{t>0}$ with $\mu_{1}=\mu$.

Proof. Let us consider an arbitrary measure $\mu \in \mathcal{I}(G)$. Since $\widehat{G}$ is supposed to be arcwise connected, $\widehat{G}$ is connected, hence its dual $G$ is aperiodic (see Hewitt and Ross [11, (24.19)]). The aperiodicity of $G$ implies that the only compact subgroup of $G$ is $\{e\}$. By the representation (6.2) we conclude that $\widehat{\mu}(\chi) \neq 0$ for all $\chi \in \widehat{G}$. From the hypotheses of the theorem it is clear that Lemma 7.1 applies and that therefore a continuous branch of $\log \widehat{\mu}$ with

$$
\log \widehat{\mu}(\mathbf{1})=0
$$

exists, where 1 denotes the identity element of the dual group which is the constant function 1. Consequently we have

$$
\left(\widehat{\mu \frac{1}{n}}(\chi)\right)^{n}=\widehat{\mu}(\chi)=\exp \{\log \widehat{\mu}(\chi)\}, \quad \chi \in \widehat{G},
$$

thus

$$
\widehat{\mu_{\frac{1}{n}}}(\chi)=\exp \left\{\frac{1}{n} \log \widehat{\mu}(\chi)\right\}, \quad \chi \in \widehat{G} .
$$


Hence the $n$-th roots $\mu_{\frac{1}{n}}$ of $\mu$ are uniquely determined. For each $r=\frac{p}{q} \in \mathbb{Q}$ with $r>0$ let

$$
\mu_{\frac{p}{q}}:=\left(\mu_{\frac{1}{q}}\right)^{p}
$$

Then

$$
\widehat{\mu \frac{p}{q}}(\chi)=\left(\exp \left\{\frac{1}{q} \log \widehat{\mu}(\chi)\right\}\right)^{p}=\exp \left\{\frac{p}{q} \log \widehat{\mu}(\chi)\right\}, \quad \chi \in \widehat{G} .
$$

If $\frac{p}{q}=\frac{p^{\prime}}{q^{\prime}} \in \mathbb{Q}$ then

$$
\widehat{\mu_{\frac{p^{\prime}}{q^{\prime}}}}(\chi)=\exp \left\{\frac{p^{\prime}}{q^{\prime}} \log \widehat{\mu}(\chi)\right\}=\exp \left\{\frac{p}{q} \log \widehat{\mu}(\chi)\right\}=\widehat{\mu \frac{p}{q}}(\chi), \quad \chi \in \widehat{G}
$$

hence the notation $\mu_{r}:=\mu_{\frac{p}{q}}$ is justified. Clearly

$$
\left(\mu_{r} * \mu_{r^{\prime}}\right)^{-}(\chi)=\widehat{\mu}_{r}(\chi) \widehat{\mu}_{r^{\prime}}(\chi)=\exp \left\{\left(r+r^{\prime}\right) \log \widehat{\mu}(\chi)\right\}=\widehat{\mu}_{r+r^{\prime}}(\chi), \quad \chi \in \widehat{G}
$$

for all $r, r^{\prime} \in \mathbb{Q}$ with $r, r^{\prime}>0$, hence $\mu_{r} * \mu_{r^{\prime}}=\mu_{r+r^{\prime}}$ for all $r, r^{\prime} \in \mathbb{Q}$ with $r, r^{\prime}>0$. Defining $\mu_{0}:=\varepsilon_{e}$, we obtain a rational convolution semigroup $\left(\mu_{r}\right)_{r \in \mathbb{Q}_{+}}$in $\mathfrak{M}^{1}(G)$.

Next we show that the mapping

$$
r \mapsto \mu_{r}
$$

from $\mathbb{Q}_{+}$into $\mathfrak{M}^{1}(G)$ is $\tau_{\mathrm{v}}{ }^{-}$and $\tau_{\mathrm{w}}$-continuous. Note that for $\mu_{n}, \nu \in \mathfrak{M}^{1}(G) \quad(n \in \mathbb{N})$ with

$$
\lim _{n \rightarrow \infty} \widehat{\mu}_{n}(\chi)=\widehat{\nu}(\chi), \quad \chi \in \widehat{G}
$$

the continuity theorem implies that

$$
\tau_{\mathrm{v}}-\lim _{n \rightarrow \infty} \mu_{n}=\nu
$$

Let $\left(r_{n}\right)_{n \geqslant 1}$ be a sequence in $\mathbb{Q}_{+}$with $r_{n} \rightarrow r \in \mathbb{Q}_{+}$. Then

$$
\widehat{\mu}_{r_{n}}(\chi)=\exp \left\{r_{n} \log \widehat{\mu}(\chi)\right\} \rightarrow \exp \{r \log \widehat{\mu}(\chi)\}=\widehat{\mu}_{r}(\chi)
$$

uniformly on compact subsets of $\widehat{G}$. This implies

$$
\tau_{\mathrm{v}}-\lim _{n \rightarrow \infty} \mu_{r_{n}}=\mu_{r}, \quad \text { hence } \quad \tau_{\mathrm{w}}-\lim _{n \rightarrow \infty} \mu_{r_{n}}=\mu_{r} .
$$

Finally we prove that $\left(\mu_{r}\right)_{r \in \mathbb{Q}_{+}}$extends to a unique $\{e\}$-continuous convolution semigroup $\left(\mu_{t}\right)_{t \in \mathbb{R}_{+}}$in $\mathfrak{M}^{1}(G)$, and hence $\mu=\mu_{1} \in \mathcal{E}(G)$. Let $r \rightarrow t \in \mathbb{R}_{+}$with $r \in \mathbb{Q}_{+}$. Then

$$
\widehat{\mu}_{r}(\chi) \rightarrow \exp \{t \log \widehat{\mu}(\chi)\}, \quad \chi \in \widehat{G} .
$$

The limiting function

$$
\chi \mapsto \exp \{t \log \widehat{\mu}(\chi)\}
$$

from $\widehat{G}$ into $\mathbb{C}$ equals 1 at $\chi=\mathbf{1}$ and is a continuous positive definite function. Hence for every $t \in \mathbb{R}_{+}$there exists a unique measure $\mu_{t} \in \mathfrak{M}^{1}(G)$ such that

$$
\widehat{\mu}_{t}(\chi)=\exp \{t \log \widehat{\mu}(\chi)\}, \quad \chi \in \widehat{G} .
$$


Clearly

$$
\left(\mu_{t} * \mu_{t^{\prime}}\right)^{\wedge}(\chi)=\widehat{\mu}_{t}(\chi) \widehat{\mu}_{t^{\prime}}(\chi)=\exp \left\{\left(t+t^{\prime}\right) \log \widehat{\mu}(\chi)\right\}=\widehat{\mu}_{t+t^{\prime}}(\chi), \quad \chi \in \widehat{G}
$$

for all $t, t^{\prime} \in \mathbb{R}_{+}$, hence $\mu_{t} * \mu_{t^{\prime}}=\mu_{t+t^{\prime}}$ for all $t, t^{\prime} \in \mathbb{R}_{+}$. The uniqueness of a continuous embedding semigroup $\left(\mu_{t}\right)_{t \in \mathbb{R}_{+}}$follows from the $\tau_{\mathrm{w}}$-continuity of the mapping

$$
r \mapsto \mu_{r}
$$

from $\mathbb{Q}_{+}$into $\mathfrak{M}^{1}(G)$.

7.4 Theorem. Let $\mu$ be an infinitely divisible probability measure on a locally compact Abelian group $G$ with arcwise connected dual group $\widehat{G}$. Then there exists a unique continuous negative definite function $\varphi: \widehat{G} \rightarrow \mathbb{C}$ such that $\varphi(\mathbf{1})=0$ and

$$
\widehat{\mu}(\chi)=\exp \{-\varphi(\chi)\}, \quad \chi \in \widehat{G} .
$$

In fact,

$$
\varphi(\chi)=-\log \widehat{\mu}(\chi), \quad \chi \in \widehat{G},
$$

and there exists an element $m \in G_{\mathcal{E}}$, a quadratic form $\psi \in \mathrm{q}_{+}(\widehat{G})$ and a Lévy measure $\eta$ on $G$ such that

$$
\varphi(\chi)=-\log \chi(m)+\psi(\chi)-\int_{G}(\chi(x)-1-i g(x, \chi)) \eta(\mathrm{d} x), \quad \chi \in \widehat{G} .
$$

7.5 Remark. The converse of this statement is obvious.

Proof. By Schoenberg's theorem (see Berg and Forst [3, p.49, Theorem 8.3]) a family $\left(\mu_{t}\right)_{t>0}$ of measures in $\mathfrak{M}^{1}(G) \quad(\mu \geqslant 0,\|\mu\| \leqslant 1)$ is an $\{e\}$-continuous convolution semigroup if and only if there exists a continuous negative definite function $\varphi: \widehat{G} \rightarrow \mathbb{C}$ such that

$$
\widehat{\mu}_{t}(\chi)=\exp \{-t \varphi(\chi)\}, \quad \chi \in \widehat{G}
$$

for all $t \in \mathbb{R}_{+}$. For $t=1$ this gives us

$$
\widehat{\mu}(\chi)=\exp \{-\varphi(\chi)\}, \quad \chi \in \widehat{G} .
$$

But $\widehat{\mu}(\mathbf{1})=1$ implies $\varphi(\mathbf{1})=0$. Since $\varphi$ is continuous, satisfies $\varphi(\mathbf{1})=0$ as well as

$$
\widehat{\mu}(\chi)=\exp \{-\varphi(\chi)\}, \quad \chi \in \widehat{G},
$$

we see that $\varphi$ is the continuous branch of $-\log \widehat{\mu}$ constructed above. The last statement follows from Theorem IV.10.1 in Parthasarathy [13].

\section{Gaussian and diffusion hemigroups}

Let $G$ be a locally compact group. 
8.1 Definition. A family $\left(\mu_{s, t}\right)_{0 \leqslant s \leqslant t}$ in $\mathfrak{M}^{1}(G)$ is called a continuous convolution hemigroup (briefly hemigroup) in $\mathfrak{M}^{1}(G)$ if $\mu_{s, r} * \mu_{r, t}=\mu_{s, t}$ for all $0 \leqslant s \leqslant r \leqslant t$, $\mu_{t, t}=\varepsilon_{e}$ for all $t \geqslant 0$, and if the mapping $(s, t) \mapsto \mu_{s, t}$ from $\mathbb{S}$ into $\mathfrak{M}^{1}(G)$ is continuous.

If $\left(\nu_{t}\right)_{t>0}$ is an $\{e\}$-continuous convolution semigroup in $\mathfrak{M}^{1}(G)$ then $\mu_{s, t}:=\nu_{t-s}$, $0 \leqslant s<t$ and $\mu_{t, t}:=\varepsilon_{e}, t \geqslant 0$ define a continuous convolution hemigroup.

Now we recall the definition of Gaussian hemigroups (see Heyer and Pap [10]).

8.2 Definition. A continuous convolution hemigroup $\left(\mu_{s, t}\right)_{0 \leqslant s \leqslant t}$ in $\mathfrak{M}^{1}(G)$ is called a Gaussian hemigroup if

$$
\lim _{Z \in \mathbf{Z}_{[0, T]}} \sum_{k=1}^{n} \mu_{\tau_{k-1}, \tau_{k}}(G \backslash N)=0
$$

for all Borel neighbourhoods $N$ of $e$ and for all $T>0$.

Corollary 2 of Theorem 2 in Siebert [15] implies that a convolution hemigroup $\left(\mu_{s, t}\right)_{0 \leqslant s \leqslant t}$ on a second countable locally compact group is a Gaussian hemigroup if and only if each associated left additive process has continuous paths with probability one.

If $G$ is a locally compact group Abelian group with countable basis of its topology then a family $\left(\mu_{s, t}\right)_{0 \leqslant s \leqslant t}$ in $\mathfrak{M}^{1}(G)$ is a Gaussian hemigroup if and only if there exist a continuous function $t \mapsto m_{t}$ from $\mathbb{R}_{+}$into $G$ with $m_{0}=e$ and a function $t \mapsto \psi_{t}$ from $\mathbb{R}_{+}$into $\mathbf{q}_{+}(\widehat{G})$ with $\psi_{0}=0$ such that $t \mapsto \psi_{t}(\chi)$ is continuous for all $\chi \in \widehat{G}$, $\psi_{t}-\psi_{s} \in \mathrm{q}_{+}(\widehat{G})$ for all $0 \leqslant s \leqslant t$, and such that

$$
\widehat{\mu}_{s, t}(\chi)=\chi\left(m_{s}^{-1} m_{t}\right) \exp \left\{-\frac{1}{2}\left(\psi_{t}(\chi)-\psi_{s}(\chi)\right)\right\} .
$$

(See Bingham [1], Heyer [6, Theorem 5.6.19] and Heyer and Pap [9]). Consequently, if $\left(\mu_{s, t}\right)_{0 \leqslant s \leqslant t}$ is a Gaussian hemigroup in $\mathfrak{M}^{1}(G)$ then $\mu_{s, t} \in \mathcal{G}_{\mathrm{P}}(G)$, i.e., $\mu_{s, t}$ is a Gaussian measure in the sense of Parthasarathy for all $0 \leqslant s \leqslant t$. Moreover, if $\left(\mu_{s, t}\right)_{0 \leqslant s \leqslant t}$ is a continuous convolution hemigroup in $\mathfrak{M}^{1}(G)$ such that $\mu_{s, t} \in \mathcal{G}_{\mathrm{P}}(G)$ for all $0 \leqslant s \leqslant t$ then $\left(\mu_{s, t}\right)_{0 \leqslant s \leqslant t}$ is a Gaussian hemigroup (see Heyer and Pap [10, 3.12]).

8.3 Examples. If $G=\mathbb{R}^{d}$ then a family $\left(\mu_{s, t}\right)_{0 \leqslant s \leqslant t}$ in $\mathfrak{M}^{1}\left(\mathbb{R}^{d}\right)$ is a Gaussian hemigroup if and only if there exist a continuous function $a: \mathbb{R}_{+} \rightarrow \mathbb{R}^{d}$ with $a(0)=0$ and a continuous function $B: \mathbb{R}_{+} \rightarrow \mathbb{R}^{d \times d}$ with $B(0)=0$ such that $B(t)-B(s)$ is symmetric positive semidefinite for all $0 \leqslant s \leqslant t$, and such that

$$
\widehat{\mu}_{s, t}\left(\chi_{y}\right)=\exp \left\{i\langle y, a(t)-a(s)\rangle-\frac{1}{2}\langle y,(B(t)-B(s)) y\rangle\right\}
$$

for all $y \in \mathbb{R}^{d} \cong\left(\mathbb{R}^{d}\right)^{\wedge}$ (see the notations in Example 5.1).

If $G=\mathbb{T}^{d}$ then a family $\left(\mu_{s, t}\right)_{0 \leqslant s \leqslant t}$ in $\mathfrak{M}^{1}\left(\mathbb{T}^{d}\right)$ is a Gaussian hemigroup if and only if there exist a continuous function $\alpha: \mathbb{R}_{+} \rightarrow[0,1)^{d}$ with $\alpha(0)=0$ and a continuous 
function $B: \mathbb{R}_{+} \rightarrow \mathbb{R}^{d \times d}$ with $B(0)=0$ such that $B(t)-B(s)$ is symmetric positive semidefinite for all $0 \leqslant s \leqslant t$, and such that

$$
\widehat{\mu}_{s, t}\left(\chi_{m}\right)=\exp \left\{2 \pi i\langle m, \alpha(t)-\alpha(s)\rangle-\frac{1}{2}\langle m,(B(t)-B(s)) m\rangle\right\}
$$

for all $m \in \mathbb{Z}^{d} \cong\left(\mathbb{T}^{d}\right)^{\wedge}$ (see the notations in Example 5.2).

If $G=\mathbb{T}^{\infty}$ then a family $\left(\mu_{s, t}\right)_{0 \leqslant s \leqslant t}$ in $\mathfrak{M}^{1}\left(\mathbb{T}^{\infty}\right)$ is a Gaussian hemigroup if and only if there exist a coordinatewise continuous function $\alpha: \mathbb{R}_{+} \rightarrow[0,1)^{\infty}$ with $\alpha(0)=0$ and an entrywise continuous function $B: \mathbb{R}_{+} \rightarrow \mathbb{R}^{\infty \times \infty}$ with $B(0)=0$ such that $B(t)-B(s)$ is symmetric positive semidefinite for all $0 \leqslant s \leqslant t$, and such that

$$
\widehat{\mu}_{s, t}\left(\chi_{m}\right)=\exp \left\{2 \pi i \sum_{k=1}^{d}(\alpha(t)-\alpha(s)) m_{k}-\frac{1}{2} \sum_{j, k=1}^{\infty}\left(b_{j, k}(t)-b_{j, k}(s)\right) m_{j} m_{k}\right\}
$$

for all $m \in \mathbb{Z}^{(\infty)} \cong\left(\mathbb{T}^{\infty}\right)^{\wedge}$ (see the notations in Example 5.3).

If $G=\Sigma_{c}$ then a family $\left(\mu_{s, t}\right)_{0 \leqslant s \leqslant t}$ in $\mathfrak{M}^{1}\left(\Sigma_{c}\right)$ is a Gaussian hemigroup if and only if there exist a continuous function $\alpha: \mathbb{R}_{+} \rightarrow[0,1)$ with $\alpha(0)=0$, a coordinatewise continuous function $j: \mathbb{R}_{+} \rightarrow \mathbb{Z}^{\infty}$ with $j(0)=0$, and an increasing continuous function $b: \mathbb{R}_{+} \rightarrow \mathbb{R}$ with $b(0)=0$ such that

$$
\begin{aligned}
\widehat{\mu}_{s, t}\left(\chi_{\gamma}\right)=\exp \{ & i\left(\alpha(t)-\alpha(s)+j_{1}(t)-j_{1}(s)+\cdots+c_{[1, k-1)}\left(j_{k-1}(t)-j_{k-1}(s)\right)\right) \gamma \\
& \left.-\frac{1}{2}(b(t)-b(s)) \gamma^{2}\right\}
\end{aligned}
$$

for all $\gamma \in\left(\Sigma_{c}\right)^{\wedge}$ (see the notations in Example 5.4). By continuity of $j$ we obtain $j(t)=0$ for all $t \geqslant 0$, hence

$$
\widehat{\mu}_{s, t}\left(\chi_{\gamma}\right)=\exp \left\{i(\alpha(t)-\alpha(s)) \gamma-\frac{1}{2}(b(t)-b(s)) \gamma^{2}\right\}
$$

for all $\gamma \in\left(\Sigma_{c}\right)^{\wedge}$. Consequently, $\mu_{s, t} \in \mathcal{G}\left(\Sigma_{c}\right)$, i.e., $\mu_{s, t}$ is a Gaussian measure for all $0 \leqslant s \leqslant t$ (not only in the wider sense of Parthasarathy). It is still an open question if this holds for all locally compact Abelian groups.

It is not easy to check whether a given continuous convolution hemigroup is Gaussian. We would like to construct Gaussian hemigroups from simpler hemigroups by deterministic change of time. We recall the definition of diffusion hemigroups (see Heyer and Pap [10]).

8.7 Definition. Let $G$ be a locally compact group. A continuous convolution hemigroup $\left(\mu_{s, t}\right)_{0 \leqslant s \leqslant t}$ in $\mathfrak{M}^{1}(G)$ is called a diffusion hemigroup if

$$
\lim _{\substack{t-s \rightarrow 0 \\ 0 \leqslant s<t \leqslant T}} \frac{1}{t-s} \mu_{s, t}(G \backslash N)=0
$$

for all Borel neighbourhoods $N$ of $e$ and for all $T>0$. 
A diffusion hemigroup is always a Gaussian hemigroup, but there exist Gaussian hemigroups on $\mathbb{R}$ which are not diffusion hemigroups (see the example in Remark 4.5 of Bingham and Heyer [4]).

8.8 Definition. A function $\lambda: \mathbb{R}_{+} \rightarrow \mathbb{R}_{+}$is called a deterministic change of time if it is strictly increasing and $\lambda\left(\mathbb{R}_{+}\right)=\mathbb{R}_{+}$. The class of deterministic changes of time will be denoted by $\Lambda$.

8.9 Remark. If $\left(\mu_{s, t}\right)_{0 \leqslant s \leqslant t}$ is a convolution hemigroup in $\mathfrak{M}^{1}(G)$ such that there exists $\lambda \in \Lambda$ with

$$
\lim _{\substack{t-s \rightarrow 0 \\ 0 \leqslant s<t \leqslant T}} \frac{1}{\lambda(t)-\lambda(s)} \mu_{s, t}(G \backslash N)=0
$$

for all Borel neighbourhoods $N$ of $e$ and for all $T>0$ then $\left(\mu_{s, t}\right)_{0 \leqslant s \leqslant t}$ is a Gaussian hemigroup. Moreover, we have

$$
\lim _{\substack{t-s \rightarrow 0 \\ 0 \leqslant s<t \leqslant T}} \frac{1}{t-s} \mu_{\lambda^{-1}(s), \lambda^{-1}(t)}(G \backslash N)=0
$$

for all Borel neighbourhoods $N$ of $e$ and for all $T>0$, hence $\left(\mu_{\lambda^{-1}(s), \lambda^{-1}(t)}\right)_{0 \leqslant s \leqslant t}$ is a diffusion hemigroup. In other words, if (8.10) holds with an appropriate $\lambda \in \Lambda$ then $\left(\mu_{s, t}\right)_{0 \leqslant s \leqslant t}$ is a deterministically time-changed diffusion hemigroup. If $G=\mathbb{R}^{d}$ then any Gaussian hemigroup is a deterministically time-changed diffusion hemigroup (see Heyer and Pap [10, 3.10]). It is still an open question whether this holds for an arbitrary locally compact Abelian group.

8.11 Remark. Let $G$ be a locally compact group with countable basis of its topology. If $\left(\nu_{t}\right)_{t \geqslant 0}$ is a Gaussian semigroup in $\mathfrak{M}^{1}(G)$ then $\mu_{s, t}:=\nu_{t-s}, 0 \leqslant s \leqslant t$ is a diffusion hemigroup. Moreover, if $\left(\nu_{t}\right)_{t \geqslant 0}$ is a convolution semigroup in $\mathfrak{M}^{1}(G)$ such that $\mu_{s, t}:=$ $\nu_{t-s}, 0 \leqslant s \leqslant t$ is a Gaussian hemigroup then $\left(\nu_{t}\right)_{t \geqslant 0}$ is a Gaussian semigroup. (See Heyer and Pap [10, 3.11].)

What hemigroups can be obtained from Gaussian semigroups by a deterministic change of time? First consider a symmetric Gaussian hemigroup $\left(\mu_{s, t}\right)_{0 \leqslant s \leqslant t}$ in $\mathfrak{M}^{1}(\mathbb{R})$. By (8.4), there exists an increasing continuous function $b: \mathbb{R}_{+} \rightarrow \mathbb{R}_{+}$with $b(0)=0$ such that

$$
\widehat{\mu}_{s, t}\left(\chi_{y}\right)=\exp \left\{-\frac{1}{2}\left((b(t)-b(s)) y^{2}\right\}, \quad \text { for all } y \in \mathbb{R} \cong(\mathbb{R})^{\wedge}\right.
$$

(see the notations in Example 5.1). Let $\left(\nu_{t}\right)_{t \geqslant 0}$ be the Gaussian semigroup in $\mathfrak{M}^{1}(\mathbb{R})$ given by

$$
\widehat{\nu}_{t}\left(\chi_{y}\right)=\exp \left\{-\frac{1}{2}(t-s) y^{2}\right\}, \quad \text { for all } y \in \mathbb{R} \cong(\mathbb{R})^{\wedge} .
$$

Then for all $0 \leqslant s \leqslant t$ and $y \in \mathbb{R} \cong(\mathbb{R})^{\wedge}$ we have $\widehat{\mu}_{s, t}\left(\chi_{y}\right)=\widehat{\nu}_{b(t)-b(s)}\left(\chi_{y}\right)$, hence $\mu_{s, t}=\nu_{b(t)-b(s)}$.

In a similar way, if $\left(\mu_{s, t}\right)_{0 \leqslant s \leqslant t}$ is a symmetric Gaussian hemigroup in $\mathfrak{M}^{1}(\mathbb{T})$ then by (8.5), there exists an increasing continuous function $b: \mathbb{R}_{+} \rightarrow \mathbb{R}_{+}$with $b(0)=0$ such that

$$
\widehat{\mu}_{s, t}\left(\chi_{m}\right)=\exp \left\{-\frac{1}{2}\left((b(t)-b(s)) m^{2}\right\}, \quad \text { for all } m \in \mathbb{Z} \cong(\mathbb{T})^{\wedge}\right.
$$


(see the notations in Example 5.2), and $\mu_{s, t}=\nu_{b(t)-b(s)}$ for all $0 \leqslant s \leqslant t$, where $\left(\nu_{t}\right)_{t \geqslant 0}$ denotes the Gaussian semigroup in $\mathfrak{M}^{1}(\mathbb{T})$ given by

$$
\widehat{\nu}_{t}\left(\chi_{m}\right)=\exp \left\{-\frac{1}{2}(t-s) m^{2}\right\}, \quad \text { for all } m \in \mathbb{Z} \cong(\mathbb{T})^{\wedge} \text {. }
$$

Moreover, if $\left(\mu_{s, t}\right)_{0 \leqslant s \leqslant t}$ is a symmetric Gaussian hemigroup in $\mathfrak{M}^{1}\left(\Sigma_{c}\right)$ then by (8.6), there exists an increasing continuous function $b: \mathbb{R}_{+} \rightarrow \mathbb{R}_{+}$with $b(0)=0$ such that

$$
\widehat{\mu}_{s, t}\left(\chi_{\gamma}\right)=\exp \left\{-\frac{1}{2}\left((b(t)-b(s)) \gamma^{2}\right\}, \quad \text { for all } \gamma \in\left(\Sigma_{c}\right)^{\wedge}\right.
$$

(see the notations in Example 5.2), and $\mu_{s, t}=\nu_{b(t)-b(s)}$ for all $0 \leqslant s \leqslant t$, where $\left(\nu_{t}\right)_{t \geqslant 0}$ denotes the Gaussian semigroup in $\mathfrak{M}^{1}\left(\Sigma_{c}\right)$ given by

$$
\widehat{\nu}_{t}\left(\chi_{\gamma}\right)=\exp \left\{-\frac{1}{2}(t-s) \gamma^{2}\right\}, \quad \text { for all } \gamma \in\left(\Sigma_{c}\right)^{\wedge} \text {. }
$$

If $b$ is strictly increasing with $\lim _{t \rightarrow \infty} b(t)=\infty$ then $b \in \Lambda$, and in each of the above cases $G=\mathbb{R}, G=\mathbb{T}$ and $G=\Sigma_{c}$ we have $\mu_{b^{-1}(s), b^{-1}(t)}=\nu_{t-s}$ for all $0 \leqslant s \leqslant t$, i.e., the Gaussian hemigroup $\left(\mu_{s, t}\right)_{0 \leqslant s \leqslant t}$ can be transformed into a Gaussian semigroup by a deterministic change of time.

In connection with the above results we mention a structural theorem that one does not find explicitly in the literature.

8.12 Theorem. Let $G$ be a locally compact Abelian group. Then the following statements are equivalent:

(i) $G$ is connected and one-dimensional;

(ii) $G$ is topologically isomorphic to $\mathbb{R}, \mathbb{T}$ or to $\Sigma_{c}$ for some $c=\left(c_{1}, c_{2}, \ldots\right) \in \mathbb{N}^{\infty}$ with $c_{k} \geqslant 2$ for all $k \in \mathbb{N}$.

For the proof we need two propositions.

8.13 Proposition. Let $H$ be a subgroup of $\mathbb{Q}, H \neq\{0\}$. Then $H$ is isomorphic to $\mathbb{Z}$ or to $\Sigma_{c}$ for some $c=\left(c_{1}, c_{2}, \ldots\right) \in \mathbb{N}^{\infty}$ with $c_{k} \geqslant 2$ for all $k \in \mathbb{N}$.

Proof. Let $P$ denote the set of all primes and let $\alpha: P \rightarrow \mathbb{Z} \cup\{-\infty\}$ be the mapping defined by

$$
\alpha(p):=\inf \left\{v_{p}(x): x \in H\right\},
$$

where $v_{p}$ signifies the multiplicity of $p$. Then (by algebraic arguments)

$$
H=\left\{x \in \mathbb{Q}: v_{p}(x) \geqslant \alpha(p) \text { for all } p \in P\right\} .
$$

We set

$$
\begin{aligned}
P_{+} & :=\{p \in P: \alpha(p)>0\}, \\
P_{0} & :=\{p \in P: \alpha(p)=0\}, \\
P_{-} & :=\{p \in P:-\infty<\alpha(p)<0\}, \\
P_{-\infty} & :=\{p \in P: \alpha(p)=-\infty\} .
\end{aligned}
$$


Since for $x \in H, x \neq 0$ and almost all $p \in P$ we have $v_{p}(x)=0, P_{+}$is finite. Let

$$
\ell:=\prod_{p \in P_{+}} p^{\alpha(p)}
$$

and

$$
M:=\left\{p^{-\alpha(p)}: p \in P_{-}\right\} \cup\left\{p^{k}: p \in P_{-\infty}, k \in \mathbb{N}\right\} .
$$

If $M$ is finite, then $P_{-\infty}=\emptyset$, and $P_{-}$is finite. In this case we put

$$
\ell^{\prime}:=\prod_{p \in P_{-}} p^{\alpha(p)}
$$

By (8.14) we obtain that $H=\ell \cdot \ell^{\prime} \cdot \mathbb{Z} \cong \mathbb{Z}$. Otherwise, let $c=\left(c_{1}, c_{2}, \ldots\right) \in \mathbb{N}^{\infty}$ be generated by counting the elements of $M$. Then, again by (8.14)

$$
H=\left\{\ell \cdot \frac{m}{c_{1} \cdot \ldots \cdot c_{n}}: m \in \mathbb{Z}, n \in \mathbb{N}\right\} \cong \Sigma_{c}
$$

and the proof is complete.

8.15 Proposition. Every torsion-free Abelian group $D$ of rank 1 is isomorphic to some subgroup of $\mathbb{Q}$.

Proof. Let $\widetilde{D}$ denote the minimal divisible extension of $D$ (Hewitt and Ross [11, A.15]). By Hewitt and Ross [11, A.16], $\widetilde{D}$ is also torsion-free of rank 1. As a torsion-free divisible group $\widetilde{D}$ is isomorphic to $\mathbb{Q}^{I}$ for some index set $I$, and from $1=\operatorname{rank} \widetilde{D}=|I|$ the assertion follows.

Proof of Theorem 8.12. (ii) $\Longrightarrow$ (i). It is clear for $\mathbb{R}$ and $T$. Since $\Sigma_{c}$ is a subgroup of $\mathbb{Q}$ and $\neq\{0\}$, hence torsion-free of rank 1, its dual $\widehat{\Sigma}_{c}$ is a compact connected (Abelian) group of dimension 1 (Hewitt and Ross [11, 25.25 and 24.28]).

(i) $\Longrightarrow$ (ii). The group $G$ is topologically isomorphic to $\mathbb{R}^{d} \times K$, where $K$ denotes a connected compact (Abelian) group. Since

$$
1=\operatorname{dim} G=\operatorname{dim}\left(\mathbb{R}^{d} \times K\right)=\operatorname{dim} \mathbb{R}^{d}+\operatorname{dim} K=d+\operatorname{dim} K
$$

(see Hofmann and Morris [12, 8.25 and 8.26]) we have either $d=1$ and $K \quad 0$-dimensional, hence connected and totally disconnected, hence $G \cong \mathbb{R}$, or $d=0$ and $K$ connected (compact, Abelian), one-dimensional. In the latter case $G \cong K$ and $\widehat{K}$ is a torsion-free discrete group of rank 1 (Hewitt and Ross [11, 24.25 and 24.28]), hence by Proposition 8.15 isomorphic to a subgroup $\neq\{0\}$ of $\mathbb{Q}$. If $\widehat{K}$ is isomorphic to $\mathbb{Z}$, then

$$
G \cong K \cong K^{\wedge \wedge} \cong \mathbb{T}
$$

(where Pontryagin's duality theorem has been applied). Otherwise, Proposition 8.13 yields

$$
K \cong K^{\wedge \wedge} \cong \widehat{\Sigma}_{c} \cong \Sigma_{c}
$$

(Hewitt and Ross $[11,25.3])$ for an appropriate $c=\left(c_{1}, c_{2}, \ldots\right) \in \mathbb{N}^{\infty}$ with $c_{k} \geqslant 2$ for all $k \in \mathbb{N}$. 
We have the conjecture that a locally compact Abelian group $G$ is one-dimensional (i.e. $G$ is isomorphic to $\mathbb{R}, T$ or to $\Sigma_{c}$ for some $c=\left(c_{1}, c_{2}, \ldots\right) \in \mathbb{N}^{\infty}$ with $c_{k} \geqslant 2$ for all $k \in \mathbb{N}$ ) if and only if all Gaussian hemigroups $\left(\mu_{s, t}\right)_{0 \leqslant s \leqslant t}$ in $\mathfrak{M}^{1}(G)$ can be represented in the form $\mu_{s, t}=\nu_{b(t)-b(s)}$ for all $0 \leqslant s \leqslant t$, where $\left(\nu_{t}\right)_{t \geqslant 0}$ denotes some Gaussian semigroup in $\mathfrak{M}^{1}(G)$ and $b: \mathbb{R}_{+} \rightarrow \mathbb{R}_{+}$is an increasing continuous function with $b(0)=0$.

8.16 Remark. Let $G$ be a locally compact group. Let $\left(\mu_{s, t}\right)_{0 \leqslant s \leqslant t}$ be a Gaussian hemigroup in $\mathfrak{M}^{1}(G)$. What extra property would garantee that $\left(\mu_{s, t}\right)_{0 \leqslant s \leqslant t}$ is a diffusion hemigroup? If $G$ is a locally compact Abelian group and $\left(\mu_{s, t}\right)_{0 \leqslant s \leqslant t}$ is Lipschitz continuous in the sense of finite variation with respect to the identity mapping then $\left(\mu_{s, t}\right)_{0 \leqslant s \leqslant t}$ is a diffusion hemigroup (see Bingham and Heyer [4, Theorem 4.1]), but this condition is too strong even in the case $G=\mathbb{R}$ (see the example in Remark 4.5 of Bingham and Heyer [4]). Our conjecture is that each Gaussian hemigroup of finite variation can be obtained as a time-changed diffusion hemigroup. (For information concerning hemigroups of finite variation see also Heyer and Pap [7], [8].)

Acknowledgments. The authors are thankful to Professors W. Hazod and W. Kaup for useful comments.

\section{References}

[1] M. S. Bingham: Stochastic processes with independent increments taking values in an Abelian group, Proc. London Math. Soc. 22 (1971), 507-530.

[2] A. Bendikov and L. Saloff-Coste: Brownian motion on compact groups in infinite dimension. To appear in: Contemporary Mathematics, Proceedings of the Heat Kernel semester, Centre Emile Borel 2002, Springer-Verlag.

[3] Ch. Berg and G. Forst: Potential Theory on Locally Compact Abelian Groups. Springer-Verlag, Berlin, Heidelberg, New York, 1975.

[4] M. S. Bingham and H. Heyer: On diffusion hemigroups of probability measures on an Abelian locally compact group. Result. Math. 37 (2000), 204-225.

[5] J. Dixmier: Quelques propriétés des groupes abéliens localement compacts. Bull. Sci. Math. II. Ser. 81 (1957), 38-48.

[6] H. Heyer: Probability Measures on Locally Compact Groups. Springer, Berlin Heidelberg New York, 1977.

[7] H. Heyer and G. PAP: Convergence of convolution hemigroups on Moore groups, in: Analysis on infinite-dimensional Lie groups and algebras, Proceedings, Luminy 1997, pp. 122-144. World Scientific, Singapore, New Jersey, London, Hong Kong, 1998.

[8] H. Heyer and G. PAP: Convergence of evolution operator families and its applications to limit theorems, Publ. Math. 58 (2001), 157-191. 
[9] H. Heyer and G. Pap: Martingale characterizations of increment processes in a locally compact group. To appear in Infinite Dimensional Analysis, Quantum Probability and Related Topics 6(3) (2003).

[10] H. Heyer and G. PAP: Gaussian hemigroups on a locally compact group. To appear in Acta Mathematica Hungarica 103(3) (2004).

[11] E. Hewitt and K. A. Ross: Abstract Harmonic Analysis. Springer-Verlag, Berlin, Göttingen, Heidelberg 1963.

[12] K. H. Hofmann and S. A. Morris: The Structure of Compact Groups. de Gruyter Studies in Mathematics 25, Berlin, 1998.

[13] K. R. Parthasarathy: Probability Measures on Metric Spaces. Academic Press, New York and London, 1967.

[14] E. SieBerT: Einbettung unendlich teilbarer Wahrscheinlichkeitsmaße auf topologischen Gruppen. Z. Wahrscheinlichkeitstheorie und verw. Gebiete 28 (1974), 227-247.

[15] E. SieBert: Jumps of stochastic processes with values in a topological group. Probab. Math. Stat. 5 (1985), 197-209.

Herbert Heyer

Mathematisches Institut

Universität Tübingen

Auf der Morgenstelle 10

D-72076 Tübingen

Germany

herbert.heyer@uni-tuebingen.de
Gyula Pap

Institute of Informatics

University of Debrecen

Pf.12

H-4010 Debrecen

Hungary

papgy@inf.unideb.hu 